\title{
MNDO Parameterized Hybrid SLG/SCF Method as Used for Molecular Modeling of Zn(II) Complexes
}

\author{
M. B. DARKHOVSKII, A. M. TOKMACHEV, A. L. TCHOUGRÉEFF \\ L. Y. Karpov Institute of Physical Chemistry, Vorontsovo Pole 10, 105064 Moscow, Russia
}

Received 29 November 2005; accepted 16 December 2005

Published online 6 February 2006 in Wiley InterScience (www.interscience.wiley.com).

DOI 10.1002/qua.20956

\begin{abstract}
The McWeeny's group functions technique is a natural way to introduce local description into quantum chemistry. It can also serve as a basis for constructing numerically effective computational schemes with almost linear scaling of computational costs with the size of a system. In this study, we apply it to the coordination compounds of $\mathrm{Zn}(\mathrm{II})$-containing ligands with nitrogen and oxygen donor atoms. In these compounds, the electron group corresponding to the metal vacant $4 s$ and $4 p$-atomic orbitals (AOs) of the $\mathrm{Zn}^{2+}$ ion and its closest coordination sphere formed by the lone pairs (LP) located on the donor atoms naturally separates. This group, designated metal and lone pairs (MLP), is described by the SCF wave function within the hybrid scheme of strictly local geminals/self-consistent field (SLG/SCF). The SLC/ SCF scheme is based on the group function approach combining different descriptions for different electron groups: two-electron two-center bonds are described by geminals (SLG groups), while all the remaining electron groups are described by Slater determinants in the one-electron (SCF) approximation (SCF groups). The original MNDO Hamiltonian parameterization for the $\mathrm{Zn}$ atom is adjusted for correct description of geometry of the complexes. (c) 2006 Wiley Periodicals, Inc. Int J Quantum Chem 106: 2268-2280, 2006
\end{abstract}

Key words: Zn(II) complexes; hybrid methods; strictly local geminals

Correspondence to: M. B. Darkhovskii; e-mail: mike@hcc.keldysh.ru

Contract grant sponsor: RFBR.

Contract grant numbers: 04-03-32146; 04-03-32206; 05-0333118; 02-07-90322. 


\section{Introduction}

M odeling of metal ion complex formation has remained a problem in computational chemistry of coordination compounds for decades [1]. Progress in modern quantum chemistry reliable predictions regarding the electronic structure and properties of polyatomic molecular systems, including metal complexes. However, high-accuracy methods, such as ab initio and density functional theory (DFT) are still computationally too demanding when dealing with coordination compounds. Qualitative approaches, such as the points-on-asphere (POS) [2] or metal-ligand size-match selectivity concept [3], still play an important role in the theoretical coordination chemistry of both transition and nontransition metals. Thus, effective numerical tools suitable for modeling of coordination bonds and reproducing their qualitative aspects are in strong demand. Recent studies reviewed in Ref. [4] are devoted to analysis of chemical bonding in various metal complexes. They are focused mainly on the covalent metal complexes with more or less isolated two-center bonds. Thus, the electronic structure of compounds considered in Ref. [4] differs on an intuitive level from the picture of unsaturable and nondirected "coordination" bonds characteristic for complexes of metals having organic ligands with donor atoms [5].

Recently a new hybrid semi-empirical method has been proposed called strictly local geminals/ self-consistent field (SLG/SCF) [6], based on the general strategy of describing molecular electronic structure in terms of electron groups residing in orthogonal carrier subspaces and each populated by integer constant numbers of electrons [7]. This method extends the original semi-empirical method [8-11] based on SLG, where the trial wave function is chosen in the form of an antisymmetrized product of SLGs. Most geminal-based approaches proposed in the literature use somehow predefined carrier spaces of one-electron states, either empirically constructed or extracted from preceding Hartree-Fock (HF) calculations by some localization procedure [12]. By contrast, employing of the special form of one-electron basis states, that is, of strictly local atomic hybrid orbitals (HOs), which are variationally determined from the energy minimum condition, is the key feature of the SLG approach [8-11]. The quantum chemical scheme [8-11] is implemented with use of semi-empirical Hamiltonians. (There are also methods employing optimized carrier spaces in the ab initio context [13-15].) The MINDO/3 [16], MNDO [17], AM1 [18], and PM3 [19] parameterizations for the molecular Hamiltonian were used. The accuracy of predicting molecular characteristics, such as heats of formation, molecular geometries [8-11], and vertical ionization potentials [20] for the SLG-based semi-empirical methods [8-11] is comparable to, and in some cases even better than, that of the corresponding SCF-based methods. The characteristic features of these methods, that is, linear scalability of required computational resources [21], correct asymptotic behavior of the electronic structure under $\sigma$-bond cleavage, and variational determination of one-electron states, make the SLGbased semi-empirical quantum chemistry an important alternative to the common SCF-based semi-empirical procedures. However, the SLGbased methods are nonuniversal, since the SLG trial wave function assumes the electronic structure of a molecule to be well depictable by a combination of two-electron chemical bonds and lone pairs. It is clear that coordination compounds do not correspond to this picture, along with many other important classes of molecular systems, such as those containing conjugated $\pi$ bonds. The extension of the SLG approach to these classes of molecular systems with essential delocalization of the electron groups is proposed in Ref. [6]. It is done within the framework of the general group function theory [7] by treating different electron groups, using different procedures: some of the groups (related to the two-electron, two-center bonds and lone pairs) are described by the SLGs; others are described by a single determinant built from molecular orbitals (MOs). This extension of the SLG approach puts the SLG/SCF method among other hybrid methods, when different parts of a molecule are treated by different methods of quantum chemistry [22].

In Ref. [6], the analysis of electronic structure, heats of formation, and geometries of a test set of organic molecules with $\pi$-electron system is given within this hybrid SLG/SCF method. The calculations show that molecular geometries and heats of formation for both conjugated double bonds and aromatic systems either formed by carbon atoms only or containing heteroatoms are reproduced with acceptable accuracy.

In the present work, we extend the approach [6] to the coordination compounds. Among these we chose for consideration the complexes of the $\mathrm{Zn}^{2+}$ ion. This choice is influenced by the facts that (i) these compounds play a significant role in chemis- 
try and especially in biochemistry, as active sites of many enzymes that contain the $\mathrm{Zn}^{2+}$ ions with different coordination numbers; and (ii) in this case there is no need to describe the $d$-shell, which is completely filled by electrons. For these reasons, the Zn(II) complexes are intensively studied by various methods of computational chemistry. There are model ab initio $\mathrm{HF}(\mathrm{MP} 2) / 6-31+\mathrm{G}^{*}$ calculations of binding enthalpy and frequencies on zinc hydrates $\mathrm{Zn}\left(\mathrm{H}_{2} \mathrm{O}\right)_{n}^{2+}$ (see Ref. [23] and references therein). Binding energies of some small complexes of $\mathrm{Zn}^{2+}$ with multiple ligands, such as acetone, $\mathrm{N}$-methylacetamide, imidazole, and water are studied within the DFT on the B3LYP/6-311++G $(d, p)$ level approximation in a series of papers [24, 25]. In one of the mentioned papers (Ref. [24]), the active site of the carbonic anhydrase enzyme is modeled by three imidazole and one water molecules, and the free energy of the complex formation is calculated. However, the study was not extended to complexes with larger ligands that characterize the common capabilities of ab initio calculations in the coordination chemistry of zinc.

The proposed sum of interactions between fragments ab initio computed (SIBFA) [26] force field has been used to predict the structure of some $\mathrm{Zn}$ (II) model complexes with small ligands, such as ammonia, water, glycine, and mercaptocarboxamides. The SIBFA methodology of empirical calculations of molecular structure is based on the fragmentation of the complex to the metal and ligands and on the modeling of electrostatic interactions between them by that of transferable multipoles and polarizabilities distributed on the atoms and bonds of the fragments precalculated with the use of an ab initio procedure [27]. The SIBFA force field was tested by comparing its energy components with $a b$ initio ones singled out by the standard energy decomposition procedure based on the "reduced variation space" decomposition [27-29]. An important feature of the SIBFA approach is that it includes an estimation of energy contribution on the electron density transfer from the lone pairs of donor atoms to the vacant orbitals of $\mathrm{Zn}^{2+}$, characteristic of the coordination bond formation.

The COSMOS force field [30] represents another approach to treating the coordination interactions. It puts an accent on accounting of the polarization of the ligands. The electron transfers are in contrast with SIBFA included implicitly through a scheme [31] employing Pauling's "bond energy-bond order" logarithmic relations [32]. The bond orders used in the COSMOS are set constants, not depen- dent on interatomic separations, so that it is not possible to treat consistently elimination of one of the coordinated ligands. The COSMOS method [33] has been applied to the calculation of some fourcoordinated complexes of zinc with an experimentally determined molecular structure [30]. However, the results of geometry and energy calculations of these "real" complexes are briefly reported in the cited work and are not compared with the experiment in detail.

The most widely used semi-empirical approaches to modeling for the latter complexes are the AM1, PM3, and MNDO/d methods. In Ref. [34], the accuracy of these three semi-empirical methods, when applied to a description of $\mathrm{Zn}$ (II) complexes, is analyzed thoroughly. It is concluded that the $\mathrm{MNDO} / \mathrm{d}$ method is the most reliable one for modeling zinc-donor atom interactions and gives more accurate results compared with the other two methods. The authors of Ref. [34] conclude that for coordination bonds of a zinc atom with the oxygen and nitrogen donor atoms, the $\mathrm{MNDO} / \mathrm{d}$ method gives results of satisfactory accuracy. However, the MNDO/d method encounters difficulties when describing complexes with polydentate ligands. This analysis was in part responsible for inspriing the development of a new "ZnB" parameterization [35] of the PM3 method for $\mathrm{Zn}$ (II) compounds in biological systems. In that paper, the heats of formation and geometry data, both from the set of [36] and the authors' [35] extensive data set obtained for some zinc metalloenzyme mimics and water complexes by the DFT (B3LYP / 6-311G*) calculations, are used for parameterization. Indeed, the resulting coordination distances and heats of formation appear to be better described by the new ZnB parameterization than by either the PM3 itself or AM1 or MNDO. (The authors of Ref. [35] did not include the MNDO/d method for the comparison of distances and heats of formation.) By contrast, the root-mean-square (RMS) errors in distances for biological mimics and water complexes are still too large: 0.08 and $0.12 \AA$, respectively, which may also indicate uncertainty of their positions as obtained from the experiment.

Taking into account that in the previous work [6] the SLG/SCF approach was tested mainly with the MNDO parameterization, we also decided to use this version of the hybrid SLG/SCF approach for calculations of $\mathrm{Zn}(\mathrm{II})$ complexes. Another reason for our choice is that the AM1 and PM3 methods seem to give artificial minima [38] at intermolecular 
separations characteristic for most of coordination complexes.

In the next section, we briefly review the general structure of the SLG/SCF hybrid method. We then apply the proposed procedure to various $\mathrm{Zn}$ (II) complexes, using an MNDO-based parameterization of the electronic Hamiltonian of the SCF part focused on the proper description of the closest coordination sphere of the metal and of $\pi$-electron subsystems of the ligands, when necessary. We then discuss the results of our modeling.

\section{Basics of the SLG/SCF Hybrid Method}

The construction of the method is based on the electron group functions formalism [7]. The electronic structure of entire molecule is represented by a set of electron groups. These groups correspond to chemical bonds, lone pairs, local unpaired valencies, and delocalized $\pi$-electron systems, or they can be constructed in other ways based on chemical intuition. The definition of any group includes the number of electrons in it and the set of one-electron basis functions spanning the corresponding carrier space. The settings for the group in the general case also include its spin state and a method (approximate form of the trial wave function) used for calculating the group wave function. The type of wave function imposes restrictions on the molecular wave function. In the semi-empirical realm, this weakness must be compensated by relevant parameterization. Nevertheless, the required modifications of the parameters, according to our experience [6], turn out to be marginal (although necessary). Below we briefly sketch the construction of the SLG/SCF approach, which is presented in detail in Ref. [6].

Construction of the hybrid orbitals (HOs) reduces to orthogonal transformations of the atomic orbitals (AOs) centered on the same atom:

$$
t_{p \sigma}^{+}=\sum_{i \in A} h_{p i}^{A} a_{i \sigma}^{+}
$$

where $a_{i \sigma}^{+}$is the creation operator of the electron with spin projection $\sigma$ on the $i$ th $\mathrm{AO},\left|t_{p \sigma}\right\rangle=t_{p \sigma}^{+}|0\rangle$ is the $\mathrm{HO}$, and the $S O(4)$ matrix $h^{A}$ determines the transformation from $\mathrm{AOs}$ to $\mathrm{HOs}$ on the atom $A$ bearing the valence $s p$-basis. The orthogonality of matrices $h^{A}$ and that of the basis AOs centered on different atoms (within the semi-empirical setting) ensures the orthogonality of the carrier spaces as- signed to the groups and, thus, the strong orthogonality of the electronic wave functions for different groups. The transformation [Eq. (1)] is meaningful only in the case of non-hydrogen atom with orbitals belonging to at least two different electron groups.

Each $\mathrm{HO}$ is uniquely assigned to some electron group. If the group is to be described by the SCF method, the molecular spin-orbitals are constructed as linear combinations of the hybrid spin-orbitals:

$$
b_{i \sigma}^{+}=\sum_{p \in\{S\}} c_{i p}^{\sigma} t_{p \sigma}^{+}
$$

where $p$ runs over the HOs forming the one-electron basis in the Sth group treated by the SCF approximation, and the expansion coefficients satisfy usual orthonormalization conditions:

$$
\sum_{p \in\{S\}} c_{i p c_{j p}^{\sigma}}^{\sigma}=\delta_{i j}
$$

Each geminal for the two-electron two-center bond is, in turn, a superposition of three singlet two-electron configurations:

$$
g_{m}^{+}=u_{m} r_{m \alpha}^{+} r_{m \beta}^{+}+v_{m} l_{m \alpha}^{+} l_{m \beta}^{+}+w_{m}\left(r_{m \alpha}^{+} l_{m \beta}^{+}+l_{m \alpha}^{+} r_{m \beta}^{+}\right),
$$

which are two ionic configurations (both electrons are on the same end of a chemical bond) and the covalent (Heitler-London type) one, respectively, representing a two-center two-electron bond (the HOs at the "right" and "left" ends of the bond are denoted $\left|r_{m}\right\rangle$ and $\left.\left|l_{m}\right\rangle\right)$. The normalization condition for the geminal amplitudes $u_{m}, v_{m}$, and $w_{m}$ reads:

$$
\left\langle 0\left|g_{m} g_{m}^{+}\right| 0\right\rangle=u_{m}^{2}+v_{m}^{2}+2 w_{m}^{2}=1 .
$$

In the case of an electronic lone pair, only one configuration survives with the amplitude equal to unity:

$$
g_{m}^{+}=r_{m \alpha}^{+} r_{m \beta}^{+} .
$$

This may be equally considered as either an SCF or an SLG function.

The wave function for the entire molecule in the SLG/SCF approximation is represented by the antisymmetrized product of molecular orbitals and geminals:

$$
|\Psi\rangle=\left(\prod_{S} \prod_{\substack{i \in\{S\} \\ \sigma}} b_{i \sigma}^{+}\right)\left(\prod_{m} g_{m}^{+}\right)|0\rangle,
$$


where index $S$ runs over all SCF-treated groups, and $i$ runs over the occupied MOs formed according to Eq. (2) from the basis states spanning the Sth SCF-treated group. Therefore, the wave function [Eq. (7)] is quite general.

The electronic Hamiltonian in the NDDO approximation is transformed as well to the basis of HOs. This transformation preserves the structure of the Hamiltonian. The expressions for molecular integrals in the $\mathrm{HO}$ basis through the transformation matrices $h^{A}$ and molecular integrals in the $\mathrm{AO}$ basis can be found in Refs. $[10,11]$. The total energy of the molecule is found by averaging the electronic Hamiltonian over the electronic wave function and adding contribution representing the core-core repulsion specific for the MNDO scheme [17].

The energy of the molecule is minimized with respect to the electronic structure variables, which are the MO LCAO coefficients $c_{i p}^{\sigma}$ [Eq. (2)], the Jacobi angles defining the $h^{A}$ matrices (and thus the shapes and directions of the HOs), and the amplitudes $u_{m}, v_{m}$ $w_{m}$ defining the geminals. An iteration scheme that alternates the HO optimizations defining the groups' carrier spaces and determinations of electron structure variables for the groups (the amplitudes of the geminals and the MO expansion coefficients, respectively) is implemented in Ref. [6]. The optimal HOs are obtained by gradient minimization of the energy with respect to sextuples of parameters determining transformation matrices $h^{A}$ [10]. The geminal amplitudes $u_{m}, v_{m}$ and $w_{m}$ are obtained by diagonalization of $3 \times 3$ matrices of effective Hamiltonians for each geminal representing a chemical bond. For determination of MOs in the SCF groups, three procedures well known in the literature [7] have been implemented in Ref. [6]: restricted Hartree-Fock (RHF), restricted open-shell Hartree-Fock (ROHF), and unrestricted Hartree-Fock (UHF). The effective Fock operators are constructed so that they take into account the presence of other electron groups. This reduces to modification of one-electron matrix elements of the effective operator.

\section{Results and Discussion}

\section{APPLICATION OF THE SLG/SCF METHOD TO ZN(II) COMPLEXES}

The hybrid method [6] briefly described in the previous section permits calculation of the electronic structure of different electron groups at different levels, effectively taking into account inter- actions between the groups. In the coordination compounds, as is shown in Ref. [42], it is natural to single out the group of electrons in the closest surrounding of the metal ion. In the considered donor complexes, the carrier space for this group of electrons is spanned by the lone pair (LP) HOs of the donor atoms and by the metal valence AOs (4s- and $4 p$-AOs, in the case of zinc). We denote the group function describing electrons in metal (M) and LPs as $\Phi_{\text {MLP. }}$. This group is to be treated by the SCF method. Other groups (pertaining to ligands) are treated either in the SCF approximation if they are $\pi$-electron systems as in benzene or pyridine rings, or in the SLG approximation if they are local $\sigma$-bonds. With these notions, the overall wave function acquires the form:

$$
\Psi=\Phi_{\mathrm{MLP}} \wedge \Phi_{\mathrm{SLG} / \mathrm{SCF}} .
$$

In the case of ligands with oxygen donor atoms, both LPs of oxygen are included in the MLP group. In molecules with the ligands bearing $\pi$-systems (e.g., imidazole, pyridine), the latter are treated as separate SCF groups without electron transfers between them.

\section{TEST CALCULATIONS}

Our main purpose was to test the effect of introducing trial wave function given by Eqs. (7) and (8) upon the structural and energy characteristics of coordination polyhedra. Thus, the re-parameterization of the MNDO Hamiltonian for the $\mathrm{Zn}$ atom with AOs included in the SCF-based group $\Phi_{\mathrm{MLP}}$ is needed to take into account the influence of the SLG-based groups. We expect the change of parameters of the SLG-based groups to be small, as for aromatic molecules bearing conjugated $\pi$-systems [6]. In the original MNDO parameterization [43] for the $\mathrm{Zn}$ atom, the parameters were fitted on a small set of di- and triatomic molecules containing zinc. A modified MNDO parameterization of zinc atom was proposed in Ref. [44], where values of twoelectron one-center parameters fitted in Ref. [43] are kept fixed; parameter $U_{s s}$ is shifted to $-17.989 \mathrm{eV}$ to obtain the heat of formation for the $\mathrm{Zn}^{2+}$ ion to be close to the experimental value of $665.1 \mathrm{kcal} / \mathrm{mol}$. (It estimates formally as $-2 U_{s s}-g_{s s}+H_{Z n}$, where the parameter $\mathrm{H}_{\mathrm{Zn}}$ is the atomization energy of $\mathrm{Zn}$ and its value accepted in MNDO is $-31.7 \mathrm{kcal} / \mathrm{mol}$; we keep it fixed.) Remaining parameters $U_{p p}, \beta_{s}, \beta_{p}$, and $\alpha$ and orbital exponents $\zeta_{s}$ and $\zeta_{p}$, were ad- 
justed. About a decade later, in Ref. [36], the MNDO/d method was introduced specifically for the $\mathrm{Zn}$ (II) compounds with another parameterization, where $U_{s s}$ as other one-electron parameters differ from those previously mentioned. Atomic parameters for zinc were modified on the basis of parameterization procedure, making use of the extended data set on compounds of $\mathrm{Zn}(\mathrm{II})$, like $\mathrm{Zn}\left(\mathrm{CH}_{3}\right)_{2}, \mathrm{Zn}(\mathrm{Acac})_{2}$, and $\mathrm{Zn}\left(\mathrm{H}_{2} \mathrm{O}\right)^{2+}$, not considered in earlier parameterizations. The $\mathrm{MNDO} / \mathrm{d}$ method gives rather accurate results for these species.

In general we accept the strategy of parameterization proprosed in Ref. [44] (see also above), but restrict ourselves to making only minimal adjustment of the parameters for the $\mathrm{Zn}$ atom to reproduce some practically interesting values, such as geometry characteristics (mainly bond lengths between $\mathrm{Zn}$ and donor atoms). It is of primary importance because there is a lot of structural information for many zinc coordination complexes; however, the experimental gas phase heats of formation for these complexes are very scarce. For that reason, some investigators $[27,35]$ use ab initio estimates for the heats of formation of other zinc complexes instead of its unknown experimental values. We try to avoid this practice, so our approach is not a parameter fitting in the strict sense.

Taking this into account and analyzing the results of Ref. [34], we started from the MNDO/d parameterization, keeping fixed the value of $U_{s s}$ as one giving the correct value for the heat of formation of the $\mathrm{Zn}^{2+}$ ion. The resonance parameters $\beta_{p}^{\mathrm{SCF}}$ of the organogenic atoms $(C, N, O, H)$ in the SLG/ SCF (MNDO) method that has already been fitted for the SCF-treated $\pi$-electron groups in Ref. [6] also retain their values. The modified MNDO $\beta_{p}^{\text {SCF }}$ parameter for the $\mathrm{N}$ atom's $\mathrm{HO}$ involved in the $\pi$-electron system was fitted in Ref. [6] to $21.97 \mathrm{eV}$. For the oxygen atom, the value of the analogous parameter $\beta_{p}^{\mathrm{SCF}}(\mathrm{O})$ fitted in Ref. [6] is $39.0 \mathrm{eV}$. (The resonance parameters were changed in the original parameterization of MNDO for the SLG method [11] itself.) These values are used in further calculations. The resonance parameter $\beta_{s}$ is tuned for the $\mathrm{N}$ and $\mathrm{O}$ donor atoms in the SCF-treated MLP group independently from other parameters mentioned above.

We then performed test calculations using the $\mathrm{MNDO}$ [43] and MNDO/d [36] parameterizations for the complexes $\mathrm{Zn}\left(\mathrm{NH}_{3}\right)_{4}^{2+}(\mathbf{1})$ and $\mathrm{Zn}(\mathrm{Im})_{6}^{2+}$ (2) (where Im = imidazole), with tetrahedral and octahedral coordination, respectively, to obtain an overall comparison of these two parameterizations of the $\mathrm{Zn}$ atom within the SLG/SCF context. It turned out that the geometry characteristics (metaldonor atom bond lengths) are better described by the MNDO/d, while the extent of charge transfer to the metal ion obtained with the MNDO parameters seems to conform better with the intuitive picture of charge redistribution in complexes giving resultant (Coulson) charge on the metal close to unity rather than to zero, which is the case for the $\mathrm{MNDO} / \mathrm{d}$ parameters. The $\mathrm{MNDO} / \mathrm{d}$ method appears to overestimate the extent of charge transfer, which may affect the resulting binding energy value. It is important, since the major contributions to the binding energy of zinc coordination compounds are electrostatic and charge transfer energies [27]. However, calculation of these complexes by the SLG/SCF method with the original MNDO/d parameters set for zinc gives good agreement between the extent of the charge transfer to the metal obtained by it and by the original MNDO. Thus, the values of the MNDO/d orbital exponents for the zinc atom $\zeta_{s}$ and $\zeta_{p}$, mostly controlling the extent of the charge transfer and the electron density distribution over $4 s-$ and $4 p$-AOs of $\mathrm{Zn}$, are concluded to be acceptable for the SLG/SCF context as well. By contrast, the geometry characteristics (bond lengths) obtained by the SLG/SCF with the $\mathrm{MNDO} / \mathrm{d}$ parameterization are in poor agreement with the experimental ones. The molecular geometry is largely influenced by the resonance parameters $\beta_{s}(\mathrm{Zn})$ and $\beta_{p}(\mathrm{Zn})$. The new values $\beta_{s}(\mathrm{Zn})=$ $1.30 \mathrm{eV}$ and $\beta_{p}(\mathrm{Zn})=1.75 \mathrm{eV}$ permit reproduction of the bond lengths in the considered complexes with the use of the SLG/SCF method with better accuracy than the MNDO/d method in its purely SCF version.

To adjust the $U_{p p}$ and $\alpha$ parameters for the $\mathrm{Zn}$ atom as well as the $\beta_{s}^{\mathrm{SCF}}$ parameter for donor atoms, we use information on geometry characteristics (bond lengths). To this end, we selected 14 complexes with ligands containing $\mathrm{N}$ and $\mathrm{O}$ donor atoms given in Table I, using the data both from Ref. [45] and from the Cambridge Crystal Structure Data Bank (CCSDB). Table I gives the ligand composition, abbreviations, and corresponding CCSDB reference codes for the selected complexes. Some less widely known ligands are depicted in Figure 1. To obtain an integral characterization of the calculated $\mathrm{Zn}$-donor atom bond lengths, we use the empirical distribution functions of the differences between the calculated and experimental bond lengths. 
TABLE I

Ligand names and Cambridge Crystal Structure DataBank (CCSDB) reference codes for the calculated molecules.

\begin{tabular}{rlll}
\hline $\mathrm{N}$ & \multicolumn{1}{c}{ Ligand composition } & Ligand abbreviation & CCSDB refcode \\
\hline 1 & Tetraamine & & Ref. \\
2 & Hexa-imidazole & $\mathrm{Im}$ & HIMZZN \\
3 & Hexaamine & & RAJNOF \\
4 & Tris(ethylenediamine) & en & CIBKON \\
5 & Bis(cis-cyclohexane-1,3,5-triamine) & chta & JUNNAH \\
6 & Bis((2-pyridylmethyl)amine) & pma & KUTNES \\
7 & Bis(1,4,7-triazacyclononane) & {$[9]$ aneN ${ }_{3}$} & KIQPAB \\
8 & Bis(diethylenetriamine) & dien & CEJYEV \\
9 & Tris(3-aminopropyl)amine & amp & BIHQOY \\
10 & Hexa-hydrate & & CAXYUV \\
11 & Hexa-methanol & MeOH & CIRJIW \\
12 & Hexa-ethanol & EtOH & [54] \\
13 & Tris(1,2-ethanediol) & diol & CIRNIA \\
14 & Bis(12-crown-4) & {$[12]$ aneO ${ }_{4}$} & ZNETD001 \\
\hline
\end{tabular}

In general, the plot of the empirical distribution function [error function, (EDF)] in the normal scale together with its linear fit is a good statistical test of the presence of systematic errors in the data set. It characterizes both the range of errors' magnitudes and the probability for that or another value of the error to appear in the test set. It is reasonable to assume that random nonsystematic errors are normally distributed with the zero mean value. Significant deviation of the EDF mean value $(\mu)$ from zero is then indicative for certain biases in the measurements or numerical experiments. The variance (or the $\sigma$ value) of this normal law characterizes the likelihood of facing an error that is too large by its absolute value. Thus, the condition for parameter $\beta_{s}^{\text {SCF }}$ fitting may be an almost zero value of $\mu$ for EDF of $\mathrm{Zn}$-donor atom bond errors in selected complexes $(<0.005 \AA)$.

The results of SLG/SCF (MNDO) calculation with the parameters $U_{p p}$ and $\alpha$ (see Table II; twoelectron one-center parameters for the zinc atom are taken from Ref. [36] without reparameterization) for the zinc atom and shifted $\beta_{s}^{\mathrm{SCF}}$ parameters for $\mathrm{N}$ and $\mathrm{O}$ donor atoms (24.0 and $36.5 \mathrm{eV}$, respectively) reasonably fulfill the mentioned requirement concerning the $\mu$ value of EDF for the zincdonor atom bond length errors. With this set of parameters, the $\mu$ values for the errors in the $\mathrm{Zn}-\mathrm{N}$ and $\mathrm{Zn}-\mathrm{O}$ bond lengths are $-0.003 \AA(\sigma=0.039$ $\AA)$ and $-0.001 \AA(\sigma=0.032 \AA)$, respectively. The standard SCF (MNDO) calculations for the same complexes result in $\mu$ values of $0.030 \AA$ ( $\sigma=0.029$
$\AA$ ) and $0.028 \AA(\sigma=0.035 \AA)$, respectively. Thus, the systematic error for the SLG/SCF (MNDO) scheme is almost zero, which can be considered an advantage in comparison with the purely SCFbased method yielding much larger systematic error. The distance variance $(\sigma)$ for SLG/SCF (MDNO) and SCF (MNDO/d) is comparable. Corresponding $\mathrm{EDF}$ and linear fit plots are given in Figures 2 and 3.

Detailed results of the geometry optimization performed by the SLG/SCF (MNDO) (with the MNDO parameterization given in Table II) and the SCF (MNDO/d) methods are presented in Table III. Although the improvement of the bond length accuracy in the case of the SLG/SCF (MNDO) method is evident as compared with the SCF $(\mathrm{MNDO} / \mathrm{d})$, the values of root-mean-square differences (RMSD) between the calculated and experimental coordination (zinc-donor atom) bond lengths in some cases are lower in the SLG/SCF (MNDO) approach. In the case of complex 10, there are two possible ways of estimating the energy: one formally using the $\mathrm{Zn}\left(\mathrm{H}_{2} \mathrm{O}\right)_{6}^{2+}$ composition of the complex, and another with the $\mathrm{Zn}\left(\mathrm{H}_{2} \mathrm{O}\right)_{18}^{2+}$ composition, reflecting the specific solvation of the hydrated cation by water molecules of the second hydration sphere, as discussed in Ref. [23]. In the first case, the $\mathrm{Zn}-\mathrm{O}$ distance is obtained to be longer by $0.01 \AA$ than in the second one and closer to the experimental value as cited in Ref. [23].

The partial atomic charges on zinc and donor atoms calculated by the SLG/SCF (MNDO) are in 


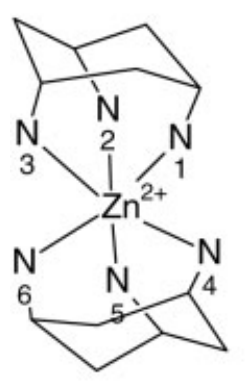

5

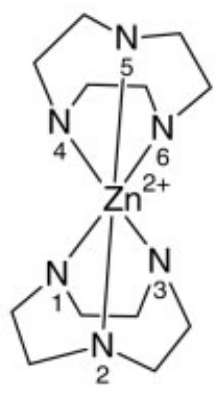

7

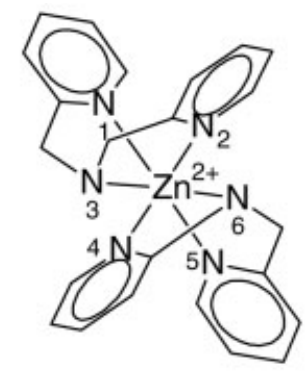

6

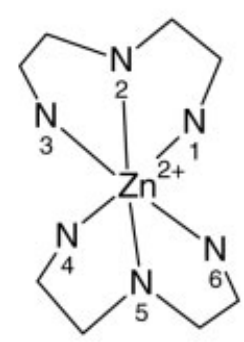

8

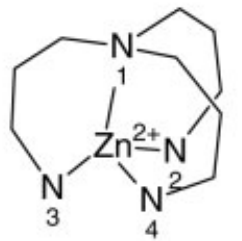

9

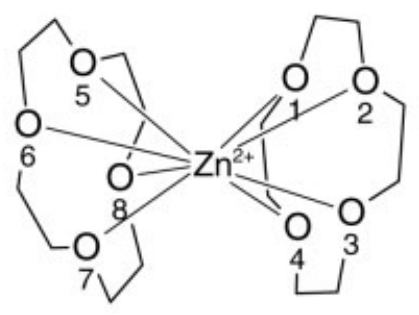

14

FIGURE 1. Ligands used in the calculations. Atom numbers correspond to Table III.

most cases larger (by magnitude) than those obtained by the SCF (MNDO/d) method. As a rule, the $\mathrm{Zn}$ effective atomic charge is $\sim 0.6 \div 0.9$ for the SLG/SCF and $0.2 \div 0.4$ for the SCF calculations. (The valence $4 s-$ and $4 p$-orbital populations of the Zn atom are, correspondingly, lower for the SLG/
TABLE II

Fitted for SLG/SCF method and initial MNDO/d Hamiltonian parameters for $\mathrm{Zn}$ atom.

\begin{tabular}{lrr}
\hline Parameter & SLG/SCF & \multicolumn{1}{c}{ SCF } \\
\hline$\zeta_{s}$ & 1.732 & 1.732 \\
$\zeta_{p}$ & 1.394 & 1.394 \\
$U_{s s}$ & -18.023 \\
$U_{p p}$ & -18.023 & -12.242 \\
$\beta_{s}$ & -10.800 & 5.017 \\
$\beta_{p}$ & 1.300 & 0.712 \\
$\alpha$ & 1.750 & 1.518 \\
\hline
\end{tabular}

SCF calculations.) Thus, the overall charge on the zinc atom obtained in the SLG/SCF (MNDO) calculations is generally close to unity, whereas in the $\mathrm{MNDO} / \mathrm{d}$ one it is rather small (from our point of view, unrealistically).

It is worthwhile to note that in the SLG/SCF method the description of coordination bonds is achieved by considering only the MLP region (electron group) around the metal atom within an SCFtreated group, which considerably reduces the overall computational costs. Comparison of calculation times for different methods can be adequately performed by estimation of the overall scaling of the applied method, i.e., dependence of calculation time on the size of the molecular system. The scaling dependence of $N^{3}$ is characteristic for

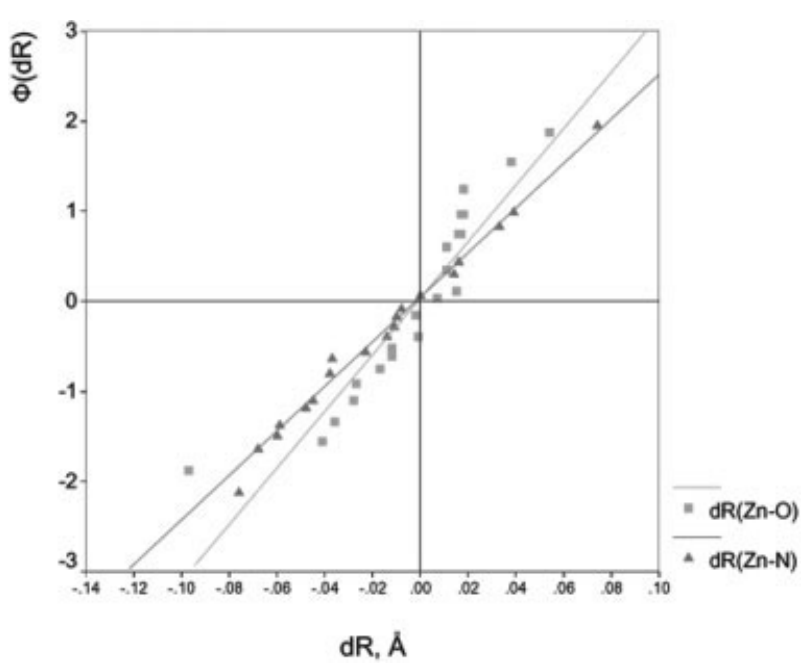

FIGURE 2. Empirical distribution function for difference in experimental bond lengths $(\AA)$ and those calculated by the SLG/SCF (MNDO) method of Zn(II) complexes with nitrogen and oxygen donor atoms. 


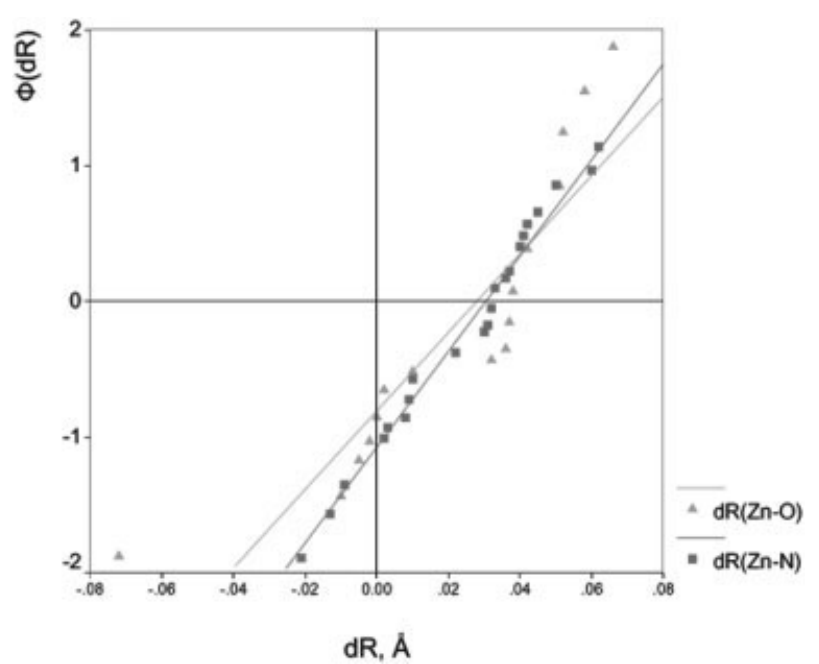

FIGURE 3. Empirical distribution function for difference in experimental bond lengths $(\AA \AA)$ and those calculated by the SCF (MNDO/d) method of $\mathrm{Zn}(\mathrm{II})$ complexes with nitrogen and oxygen donor atoms.

pure SCF procedure in standard semi-empirical methods, whereas for polarizable force fields such as SIBFA it scales as $N^{2}$. The current implementation of the SLG/SCF (MNDO) method scales as $N^{2}$ similar to SIBFA, for both the energy and gradient calculations. Unfortunately, due to transformation of two-electron integrals from the $\mathrm{AO}$ to $\mathrm{HO}$ basis, this dependence has large proportionality coefficient. The use of expansion over multipoles in the two-electron two-center integrals permits appreciable reduction of this coefficient [21]. Furthermore, use of strictly local orbitals allows us to cut off interactions between distant atoms effectively, which results in almost linear scaling of the SLG (as well as SLG/SCF) procedure [21], provided all SCF groups contain a small number of electrons, so the number of the electron groups grows linearly with that of the size of the system. This difference in scaling should be mostly manifested for calculations of high-molecular zinc-containing enzymes.

\section{EFFECTS OF LIGAND SUBSTITUTION: NUMERICAL EXPERIMENT}

The effects of substitution of the ligands in the coordination sphere of the $\mathrm{Zn}^{2+}$ complexes were also of interest to us. The qualitative theory of metal complex geometry is given in Ref. [46]. Model considerations [46] of the substitution in octahedral complexes are based on perturbative treatment of variation of diagonal matrix element of a model
Fock operator describing the close ligand shell of a complex. Within the SLG/SCF treatment for the complexes introduced in the present work, the corresponding Fock operator for the MLP electron group appears naturally, and its matrix elements can be evaluated directly.

We consider substitution in six-coordinated 12electron (according to specification of [46]) complexes of the form $\mathrm{ZnL}_{5} \mathrm{X}$. In the case of the $\mathrm{ZnL}_{6}$ complex, the frontier MOs have the form:

$$
\begin{aligned}
& \text { HOMO: } \psi\left(e_{g}\right)=(1 / \sqrt{12})\left(2 \sigma_{1}\right. \\
& \left.+2 \sigma_{2}-\sigma_{3}-\sigma_{4}-\sigma_{5}-\sigma_{6}\right) \\
& \text { LUMO: } \begin{aligned}
\psi\left(a_{1 g}\right)=l_{a} s- & \left(c_{a} / \sqrt{6}\right)\left(\sigma_{1}\right. \\
+ & \left.\sigma_{2}+\sigma_{3}+\sigma_{4}+\sigma_{5}+\sigma_{6}\right), \quad(9)
\end{aligned}
\end{aligned}
$$

where $\sigma_{i}$ stands for the $i$ th $\sigma$-LPs included in the MLP group. In this setting, the theory [46] predicts that for symmetry reasons only the normal mode corresponding to tetragonal distortion of the complex is capable these frontier MOs (in the first order of perturbation theory), so only this distortion can take place as a result of the substitution. According to the theory [46], in this case the amplitude $Q$ of the normal mode responding to the substitution is the product of electronic and vibronic factors:

$$
Q=-\left.\frac{1}{3 \sqrt{2}} c_{a} l_{a} \delta F_{X L} \frac{\partial F_{s L}}{\partial R}\right|_{R=R_{0}} .
$$

In Eq. (10), coefficients $c_{a}$ and $l_{a}$ are taken from Eq. (9), $\delta F_{X L}$ is the difference of the diagonal matrix elements of the group MLP effective Fock operator for the LP of the substituent $X$ and for that of the predominant ligand $L, \delta F_{X L}=F_{X X}-F_{L L}$ (electronic factor), and the last term contains the derivative of the off-diagonal Fock operator matrix element $F_{s L}$ between lone pair $L$ and $4 s-\mathrm{AO}$ of the zinc atom with respect to the metal-donor atom bond length $R$ at its value $R_{0}$ in the initial complex $\mathrm{ZnL}_{6}$ (vibronic factor). In the theory [46], the latter multiplier is assumed to be always positive. This is supported by our calculations. Thus, the sign of $Q$ depends only on that of $\delta F_{X L}$, since the expansion coefficients $c_{a}, l_{a}$ are chosen to be positive. According to Ref. [46], the effect of the substituent in octahedral $12 \bar{e}$ complexes manifests itself in shortening of the bond in the trans-position with respect to the substituted position if the substituent $X$ is a stronger donor than the substituted ligand $L$. The 
TABLE III

Lengths and RMSD of coordination bonds, calculated from structures of the investigated complexes optimized by the SLG/SCF (MNDO) and SCF (MNDO/d) methods.*

\begin{tabular}{|c|c|c|c|c|c|c|c|c|c|}
\hline \multirow{2}{*}{$\begin{array}{l}\text { Complex } \\
\text { no. }\end{array}$} & \multirow[b]{2}{*}{ Bond } & \multicolumn{3}{|c|}{ Bond lengths $(\AA)$} & \multirow{2}{*}{$\begin{array}{c}\text { Complex } \\
\text { no. }\end{array}$} & \multirow[b]{2}{*}{ Bond } & \multicolumn{3}{|c|}{ Bond lengths $(\AA)$} \\
\hline & & SLG/SCF & SCF & exp. & & & SLG/SCF & SCF & exp. \\
\hline \multirow[t]{5}{*}{1} & $\mathrm{Zn}-\mathrm{N}$ & 2.042 & 2.085 & 2.052 & \multirow[t]{5}{*}{$8^{a}$} & $\mathrm{~N} 1$ & 2.176 & 2.215 & 2.219 \\
\hline & $\mathrm{Zn}-\mathrm{N}$ & 2.041 & 2.083 & 2.052 & & $\mathrm{~N} 2$ & 2.188 & 2.226 & 2.163 \\
\hline & $\mathrm{Zn}-\mathrm{N}$ & 2.041 & 2.084 & 2.052 & & N3 & 2.177 & 2.224 & 2.230 \\
\hline & $\mathrm{Zn}-\mathrm{N}$ & 2.041 & 2.084 & 2.052 & & N4 & 2.170 & 2.224 & 2.245 \\
\hline & RMSD & 0.011 & 0.032 & - & & N5 & 2.188 & 2.227 & 2.155 \\
\hline \multirow{7}{*}{2} & & & & & \multirow{7}{*}{$9^{a}$} & N6 & 2.165 & 2.215 & 2.204 \\
\hline & $\mathrm{Zn}-\mathrm{N}$ & 2.213 & 2.232 & 2.180 & & RMSD & 0.048 & 0.040 & - \\
\hline & $\mathrm{Zn}-\mathrm{N}$ & 2.216 & $\begin{array}{l}2.263 \\
2\end{array}$ & $\begin{array}{l}2.200 \\
2200\end{array}$ & & $\mathrm{~N} 1$ & 2.037 & 2.080 & 1.992 \\
\hline & $\mathrm{Zn}-\mathrm{N}$ & 2.214 & 2.227 & $\begin{array}{l}2.200 \\
2.180\end{array}$ & & N2 & 2.035 & 2.080 & 1.983 \\
\hline & $\begin{array}{l}\mathrm{Zn}-\mathrm{N} \\
\mathrm{Zn}-\mathrm{N}\end{array}$ & $\begin{array}{l}2.213 \\
2.216\end{array}$ & $\begin{array}{l}2.236 \\
2.238\end{array}$ & $\begin{array}{l}2.180 \\
2.200\end{array}$ & & N3 & 2.094 & 2.110 & 2.097 \\
\hline & $\mathrm{Zn}-\mathrm{N}$ & 2.214 & 2.242 & 2.200 & & N4 & 2.033 & 2.070 & 2.001 \\
\hline & RMSD & 0.023 & 0.048 & - & & RMSD & 0.038 & 0.074 & - \\
\hline \multirow{8}{*}{3} & & & & & \multirow[t]{7}{*}{10} & $\mathrm{Zn}-\mathrm{O}$ & 2.057 & 2.066 & 2.052 \\
\hline & $\mathrm{Zn}-\mathrm{N}$ & 2.169 & 2.215 & 2.207 & & $\mathrm{Zn}-\mathrm{O}$ & 2.067 & 2.086 & 2.049 \\
\hline & $\mathrm{Zn}-\mathrm{N}$ & 2.169 & 2.216 & 2.207 & & $\mathrm{Zn}-\mathrm{O}$ & 2.067 & 2.087 & 2.049 \\
\hline & $\mathrm{Zn}-\mathrm{N}$ & 2.170 & 2.217 & 2.207 & & $\mathrm{Zn}-\mathrm{O}$ & 2.067 & 2.086 & 2.049 \\
\hline & $\mathrm{Zn}-\mathrm{N}$ & 2.169 & 2.217 & 2.207 & & $\mathrm{Zn}-\mathrm{O}$ & 2.066 & 2.086 & 2.049 \\
\hline & $\mathrm{Zn}-\mathrm{N}$ & 2.169 & 2.216 & 2.207 & & $\mathrm{Zn}-\mathrm{O}$ & 2.066 & 2.087 & 2.049 \\
\hline & $\mathrm{Zn}-\mathrm{N}$ & 2.170 & 2.216 & 2.207 & & RMSD & 0.017 & 0.037 & - \\
\hline & RMSD & 0.035 & 0.009 & - & \multirow[t]{7}{*}{11} & $\mathrm{Zn}-\mathrm{O}$ & 2.097 & 2.128 & 2.086 \\
\hline \multirow[t]{7}{*}{4} & $\mathrm{Zn}-\mathrm{N}$ & 2.166 & 2.217 & 2.214 & & $\mathrm{Zn}-\mathrm{O}$ & 2.097 & 2.128 & 2.086 \\
\hline & $\mathrm{Zn}-\mathrm{N}$ & 2.168 & 2.215 & 2.213 & & $\mathrm{Zn}-\mathrm{O}$ & 2.097 & 2.128 & 2.086 \\
\hline & $\mathrm{Zn}-\mathrm{N}$ & 2.169 & 2.220 & 2.229 & & $\mathrm{Zn}-\mathrm{O}$ & 2.097 & 2.128 & 2.086 \\
\hline & $\mathrm{Zn}-\mathrm{N}$ & 2.165 & 2.220 & 2.241 & & $\mathrm{Zn}-\mathrm{O}$ & 2.097 & 2.128 & 2.086 \\
\hline & $\mathrm{Zn}-\mathrm{N}$ & 2.170 & 2.220 & 2.229 & & $\mathrm{Zn}-\mathrm{O}$ & 2.097 & 2.128 & 2.086 \\
\hline & $\mathrm{Zn}-\mathrm{N}$ & 2.165 & 2.220 & 2.233 & & RMSD & 0.000 & 0.042 & - \\
\hline & RMSD & 0.060 & 0.011 & - & \multirow[t]{7}{*}{12} & $\mathrm{Zn}-\mathrm{O}$ & 2.077 & 2.131 & 2.079 \\
\hline \multirow[t]{8}{*}{$5^{a}$} & N1 & 2.179 & 2.228 & 2.187 & & $\mathrm{Zn}-\mathrm{O}$ & 2.077 & 2.130 & 2.079 \\
\hline & N2 & 2.182 & 2.227 & 2.182 & & $\mathrm{Zn}-\mathrm{O}$ & 2.078 & 2.130 & 2.079 \\
\hline & N3 & 2.183 & 2.228 & 2.191 & & $\mathrm{Zn}-\mathrm{O}$ & 2.077 & 2.131 & 2.079 \\
\hline & N4 & 2.182 & 2.228 & 2.192 & & $\mathrm{Zn}-\mathrm{O}$ & 2.077 & 2.130 & 2.079 \\
\hline & N5 & 2.180 & 2.227 & 2.194 & & $\mathrm{Zn}-\mathrm{O}$ & 2.078 & 2.130 & 2.079 \\
\hline & N6 & 2.183 & 2.228 & 2.206 & & RMSD & 0.002 & 0.051 & - \\
\hline & RMSD & 0.013 & 0.036 & - & \multirow[t]{7}{*}{13} & $\mathrm{Zn}-\mathrm{O}$ & 2.074 & 2.088 & 2.086 \\
\hline & & & & & & $\mathrm{Zn}-\mathrm{O}$ & 2.070 & 2.088 & 2.098 \\
\hline \multirow[t]{7}{*}{$6^{a}$} & N1 & 2.175 & 2.191 & 2.159 & & $\mathrm{Zn}-\mathrm{O}$ & 2.072 & 2.089 & 2.089 \\
\hline & N2 & 2.225 & 2.193 & 2.151 & & $\mathrm{Zn}-\mathrm{O}$ & 2.074 & 2.088 & 2.086 \\
\hline & N3 & 2.197 & 2.220 & 2.158 & & $\mathrm{Zn}-\mathrm{O}$ & 2.070 & 2.088 & 2.098 \\
\hline & N4 & 2.175 & 2.191 & 2.159 & & $\mathrm{Zn}-\mathrm{O}$ & 2.072 & 2.089 & 2.089 \\
\hline & N5 & 2.225 & 2.193 & 2.151 & & RMSD & 0.020 & 0.006 & - \\
\hline & N6 & 2.197 & 2.220 & 2.158 & \multirow{2}{*}{$14^{\mathrm{b}}$} & $\mathrm{Zn}-\mathrm{O} 1$ & 2.259 & 2.281 & 2.244 \\
\hline & RMSD & 0.049 & 0.047 & - & & $\mathrm{Zn}-\mathrm{O} 2$ & $\begin{array}{l}2.259 \\
2.258\end{array}$ & 2.278 & 2.220 \\
\hline $7^{a}$ & $\mathrm{~N} 1$ & 2.167 & 2.211 & 2.182 & & $\mathrm{Zn}-\mathrm{O} 3$ & 2.243 & 2.277 & 2.279 \\
\hline & N2 & 2.173 & 2.212 & 2.166 & & $\mathrm{Zn}-\mathrm{O} 4$ & 2.268 & 2.280 & 2.214 \\
\hline & N3 & 2.177 & 2.212 & 2.173 & & $\mathrm{Zn}-\mathrm{O} 5$ & 2.241 & 2.277 & 2.282 \\
\hline & N4 & 2.168 & 2.211 & 2.182 & & $\mathrm{Zn}-\mathrm{O} 6$ & 2.256 & 2.281 & 2.249 \\
\hline & N5 & 2.173 & 2.212 & 2.166 & & $\mathrm{Zn}-\mathrm{O} 7$ & 2.242 & 2.279 & 2.269 \\
\hline & N6 & 2.178 & 2.212 & 2.173 & & $\mathrm{Zn}-\mathrm{O} 8$ & 2.252 & 2.277 & 2.349 \\
\hline & RMSD & 0.010 & 0.039 & - & & RMSD & 0.036 & 0.041 & - \\
\hline
\end{tabular}

* Experimental geometry references can be found in Table I.

${ }^{\text {a }}$ For atomic numbers, see Fig. 1.

${ }^{\mathrm{b}}$ For atomic numbers, see Fig. 1. 
donor strength in its turn can be identified with the magnitude of the diagonal matrix element of the Fock operator over its lone pair $\mathrm{HO}$ and a stronger donor is that whose matrix element is less negative.

We performed calculations with use of the SLG/ SCF (MNDO) model on several characteristic examples. First, the hexa-imidazole zinc complex 2 is considered, where one imidazole ligand is substituted for ammonia, resulting in the $\mathrm{Zn}(\mathrm{Im})_{5}\left(\mathrm{NH}_{3}\right)^{2+}$ complex. So, $L=\mathrm{Im}, X=\mathrm{NH}_{3}$, the value of $F_{L L}=$ $-23.95 \mathrm{eV}, F_{X X}=-23.02 \mathrm{eV}$, and the difference $\delta F_{X L}$ is $0.93 \mathrm{eV}$, so ammonia is a stronger donor than imidazole. The effect must be manifested by the bond shortening for the N6 atom in the transposition to the incoming ammonia molecule. This is perfectly reproduced in our calculations (see Table IV). The extent of the observed substitution effect on the bond lengths for N3 and N6 atoms is $\sim 0.06$ $\AA$, and the variation of the coordination bond lengths under substitution is almost ideally symmetric.

Then, we consider hexa-amino-zinc complex 3, where one ammonia ligand is substituted for the pyridine ligand, resulting in the $\mathrm{Zn}\left(\mathrm{NH}_{3}\right)_{5}(\mathrm{py})^{2+}$ complex. In this complex $L=\mathrm{NH}_{3}, X=$ py, and in the initial configuration corresponding to the un- substituted complex the matrix element $F_{L L}=$ $-24.22 \mathrm{eV}$, and $F_{X X}=-24.76 \mathrm{eV}$, so the difference between them is $\delta F_{X L}=-0.54 \mathrm{eV}$. Thus, the variation of this diagonal matrix element is negative, and the pyridine is a weaker donor than ammonia. As one can see from Table IV, the theoretically expected effect on geometry is reproduced, and the trans-ammonia ligand and the substituent pyridine both go away from the zinc atom. The magnitude of elongation of the coordination bond lengths under substitution is smaller than that in the complex 2 and nonsymmetrical, i.e., $0.044 \AA$ and $0.020 \AA$ for the N3 and N6 atoms, respectively.

As the third example, one pyridine ligand in the hypothetical hexapyridine zinc complex $\mathrm{Zn}(\mathrm{py})_{6}^{2+}$ is substituted for the linear ligand acetonitrile $\mathrm{CH}_{3} \mathrm{CN}$ to obtain $\mathrm{Zn}(\mathrm{py})_{5}\left(\mathrm{CH}_{3} \mathrm{CN}\right)^{2+}$. Here $\mathrm{L}=\mathrm{py}$, $\mathrm{X}=\mathrm{CH}_{3} \mathrm{CN}, F_{L L}=-23.95 \mathrm{eV}, F_{X X}=-26.61 \mathrm{eV}$, $\delta F_{X L}=-2.7 \mathrm{eV}$, and the sign of the difference $\delta F_{X L}$ is negative; thus, acetonitrile is a weaker donor ligand. The variations of the coordination bond lengths in $\mathrm{Zn}(\mathrm{py})_{5}\left(\mathrm{CH}_{3} \mathrm{CN}\right)^{2+}$, as compared with $\mathrm{Zn}(\mathrm{py})_{6}^{2+}$, are $-0.22 \AA$ and $-0.1 \AA$ for acetonitrile and trans-pyridine, respectively, and become shorter, contradicting the simplest variant of the theory [46]. We also see that the two distances

TABLE IV

Substitution effects in bond lengths and dissociation energy $D, \Delta D=D\left(Z n L_{5} X\right)-D\left(Z_{n} L_{6}\right)$, for selected complexes calculated by the SLG/SCF (MNDO) method.

\begin{tabular}{|c|c|c|c|c|}
\hline \multirow[b]{2}{*}{ Complex } & \multirow[b]{2}{*}{$\Delta D(\mathrm{kcal} / \mathrm{mol})$} & \multirow[b]{2}{*}{ Donor atom } & \multicolumn{2}{|c|}{ Bond length $(\AA)$} \\
\hline & & & Substituted & Initial \\
\hline \multirow[t]{6}{*}{$\mathrm{Zn}(\mathrm{Im})_{5}\left(\mathrm{NH}_{3}\right)^{2+}$} & -4.1 & $\mathrm{~N} 1(\mathrm{X})$ & 2.235 & 2.213 \\
\hline & & N2(Y) & 2.222 & 2.216 \\
\hline & & N3(Amm)(Z) & 2.156 & 2.214 \\
\hline & & $\mathrm{N} 4(-\mathrm{X})$ & 2.231 & 2.213 \\
\hline & & N5(-Y) & 2.226 & 2.216 \\
\hline & & N6(-Z) & 2.156 & 2.214 \\
\hline \multirow[t]{6}{*}{$\mathrm{Zn}\left(\mathrm{NH}_{3}\right)_{5}(\mathrm{Py})^{2+}$} & 4.4 & $\mathrm{~N} 1(\mathrm{X})$ & 2.165 & 2.169 \\
\hline & & N2(Y) & 2.165 & 2.169 \\
\hline & & N3(Py)(Z) & 2.214 & 2.170 \\
\hline & & $\mathrm{N} 4(-\mathrm{X})$ & 2.165 & 2.169 \\
\hline & & N5(-Y) & 2.166 & 2.169 \\
\hline & & N6(-Z) & 2.190 & 2.170 \\
\hline \multirow[t]{6}{*}{$\mathrm{Zn}(\mathrm{Py})_{5}\left(\mathrm{CH}_{3} \mathrm{CN}\right)^{2+}$} & -16.4 & $\mathrm{~N} 1(\mathrm{X})$ & 2.282 & 2.288 \\
\hline & & N2(Y) & 2.323 & 2.288 \\
\hline & & $\mathrm{N} 3\left(\mathrm{CH}_{3} \mathrm{CN}\right)(\mathrm{Z})$ & 2.079 & 2.285 \\
\hline & & $\mathrm{N} 4(-\mathrm{X})$ & 2.286 & 2.288 \\
\hline & & N5(-Y) & 2.321 & 2.288 \\
\hline & & N6(-Z) & 2.181 & 2.285 \\
\hline
\end{tabular}


change to a different extent. The reasons for the mentioned disagreement may be various. First, we mention that the difference from the results of the theory [46] occurs when somewhat bulkier ligands are involved and can at least partially be attributed to some steric effects. In contrast, predictions of the simplest version of the theory [46] can be modified when going to its more refined variant involving other orbitals in addition to the frontier ones. For example, another normal mode, involving antisymmetric displacement of the trans-ligands can be populated. Finally, we note that the nitrile substitution results in a much stronger validation of the diagonal Fock matrix element than in other cases, so that the perturbative treatment of Ref. [46] may become invalid.

\section{Conclusion}

In the present work, we performed parameterization of a recently proposed hybrid SLG/SCF method at the MNDO level to calculate heats of formation and equilibrium geometries of $\mathrm{Zn}$ (II) complexes with ligands containing nitrogen and and oxygen donor atoms. It is shown that the proposed method is capable of reproducing with remarkable accuracy the geometry characteristics of the complexes. The method takes into account the influence of the metal ion upon electronic structure of the ligands both by polarization and by charge transfer. The latter is responsible for chemical bonding and thus for the metal complex formation. The standard ab initio and DFT techniques provide no specialized approach to the description of the so-called coordination bonds [5], characterized by principal unsaturability and flexibility of geometry orientations and placement of donor atoms around metal cation. As compared with empirical approaches taking into account the charge redistribution throughout the complex formation (e.g., SIBFA and COSMOS force fields), the proposed one uses a sequential quantum chemical procedure to model the electron redistribution. The results of the calculations on substituted complexes are in fair agreement with the qualitative theory of the ligand influence in complexes. The usage of inherently local description for one-electron states of the complex may be helpful for developing, in the future, a consistent form of the junctions to the surrounding treated by molecular mechanics methods that may be relevant for modeling metal-containing enzymes.

\section{ACKNOWLEDGMENTS}

This work is dedicated to Professor A. A. Levin on the occasion of his 75th birthday. The authors gratefully acknowledge valuable discussions with Dr. I. V. Pletnev and Dr. V. V. Zernov. Usage of the CCSDB is supported by the RFBR.

\section{References}

1. Hancock, R. Prog Inorg Chem 1989, 37, 187.

2. Kepert, D. Inorganic Stereochemistry; Springer: Berlin, 1982.

3. Hay, B. P.; Hancock, R. D. Coord Chem Rev 2001, 212, 61.

4. Frenking, G.; Fröhlich, N. Chem Rev 2000, 100, 717.

5. Bersuker, I. B. Electronic Structure and Properties of Transition Metal Compounds: Introduction to the Theory; John Wiley \& Sons: New York, 1996.

6. Tokmachev A. M.; Tchougréeff, A. L. Int J Quantum Chem 2006, 106, 571.

7. McWeeny, R. Methods of Molecular Quantum Mechanics; 2nd ed.; Academic Press: London, 1992.

8. Tokmachev, A. M.; Tchougréeff, A. L. Russ J Phys Chem 1999, 73, 320.

9. Tokmachev, A. M.; Tchougréeff, A. L.; Misurkin I. A. Russ J Phys Chem 2000, 74 (Suppl 2), S205.

10. Tokmachev, A. M.; Tchougréeff, A. L. J Comp Chem 2001, 22,752 .

11. Tokmachev, A. M.; Tchougréeff, A. L. J Phys Chem A 2003, $107,358$.

12. Surján, P. R. In Correlation and Localization; Vol 203, Topics in Current Chemistry; Surján, P. R., Ed.; Springer-Verlag: Berlin, 1999; p 165.

13. Li, J. B.; McWeeny, R. Int J Quantum Chem 2002, 89, 208.

14. Rassolov, V. A. J Chem Phys 2002, 117, 5978.

15. Tokmachev, A. M.; Dronskowski, R. J Comp Chem 2006, 27, 296.

16. Bingham, R. C.; Dewar, M. J. S.; Lo, D. H. J Am Chem Soc 1975, 97, 1302.

17. Dewar, M. J. S.; Thiel, W. J Am Chem Soc 1977, 99, 4907.

18. Dewar, M. J. S.; Zoebisch, E. G.; Healy, E.; Stewart, J. J. P. J Am Chem Soc 1985, 107, 3902.

19. Stewart, J. J. P. J Comp Chem 1989, 10, 209.

20. Tokmachev, A. M.; Tchougréeff, A. L.; Misurkin, I. A. Int J Quantum Chem 2001, 83, 109.

21. Tokmachev, A. M.; Tchougréeff, A. L. J Phys Chem A 2005, $109,7613$.

22. Tchougréeff, A. L.; Tokmachev, A. M. In Advanced Topics in Theoretical Chemical Physics; Vol 12, Progress in Theoretical Chemistry and Physics; Maruani, J.; Lefebvre, R.; Brändas, E., Eds.; Kluwer: Dordrecht, 2003; p 207.

23. Rudolph, W. W.; Pye, C. C. Phys Chem Chem Phys 1999, 1, 4583.

24. Peschke, M.; Blades, A. T.; Kebarle, P. J Am Chem Soc 2000, $122,1492$.

25. Peschke, M.; Blades, A. T.; Kebarle, P. J Am Chem Soc 2000, $122,10440$. 
26. Gresh, N.; Claverie, P.; Pullman, A. Int J Quantum Chem 1986, 29, 101.

27. Tiraboschi, G.; Gresh, N.; Giessner-Prette, C.; Pedersen, L.; Deerfield, D. J Comp Chem 2000, 21, 1011

28. Rogalewicz, F.; Ohanessian, G.; Fresh, N. J Comp Chem 2000, 21, 963.

29. Tiraboschi, G.; Fournié-Zaluski, M. C.; Roques, B. P.; Gresh, N. J Comp Chem 2001, 22, 1038.

30. Sternberg, U.; Koch, F. T.; Bräuer, M.; Kunert, M.; Anders, E. J Mol Model 2001, 7, 54.

31. OKeefe, M.; Brese, N. E. J Am Chem Soc 1991, 113, 3226.

32. Pauling, L. The Nature of the Chemical Bond; Cornell University Press: Ithaca, NY, 1960.

33. Möllhoff, M.; Sternberg, U. J Mol Model 2001, 7, 90.

34. Bräuer, M.; Kunert, M.; Dinjus, E.; Klussmann, M.; Döring, M.; Görls, H.; Anders, E. J Mol Struct (Theochem) 2000, 505, 289.

35. Brothers, E. N.; Suarez, D.; Deerfield, D. W., II; Merz, K. M., Jr. J Comp Chem 2004, 25, 1677.

36. Thiel, W.; Voityuk, A. A. J Phys Chem 1996, 100, 616.

37. Kilshtain-Vardi, A.; Shoham, G.; Goldblum, A. Int J Quantum Chem 2002, 88, 87.

38. Csonka, G.; Ángyán, J. J Mol Struct (Theochem) 1997, 393, 31.

39. Surján, P. Second Quantized Approach to Quantum Chemistry; Springer: Berlin, 1989.

40. Jørgensen, P.; Simons, J. Second Quantization-Based Methods in Quantum Chemistry; Academic Press: New York, 1981.

41. Hamermesh, M. Group Theory and Its Applications in Physics; Dover Publications, Inc.: New York, 1965.

42. Tchougréeff, A. L. J Mol Struct (Theochem) 2003, 632, 91.
43. Dewar, M. J. S.; Merz, K. M. Organometallics 1986, 5, 1494.

44. Voityuk, A. A.; Bliznyuk, A. A. Zh Strukt Khim 1987, 28, 20 [in Russian].

45. Mel'nikov, V. L.; Pletnev, I. V. Izv Akad Nauk Ser Khim 1997, 7, 1278 [in Russian].

46. Levin, A. A.; Dyachkov, P. N. Heteroligand Molecular Systems: Bonding, Shapes, and Isomer Stabilities; Taylor \& Francis: New York, 2002.

47. Guggenberger, L. J. Inorg Chem 1969, 8, 2771.

48. Sandmark, C.; Branden, C. Acta Chem Scand 1967, 21, 993.

49. Brumm, H.; Jansen, M. Z Anorg Allg Chem 2001, 627, 1433.

50. Cernak, J.; Chomic, J.; Dunaj-Jurco, M.; Kappenstein, C. Inorg Chim Acta 1984, 85, 219.

51. Marsh, R. E.; Kapon, M.; Hu, S.; Herbstein, F. H. Acta Crystallogr B 2002, 58, 62.

52. Glerup, J.; Goodson, P. A.; Hodgson, D. J.; Michelsen, K.; Nielsen, K. M.; Weihe, H. Inorg Chem 1992, 31, 4611.

53. Kavana, M.; Powell, D. R.; Burstyn, J. N. Inorg Chim Acta 2000, 297, 351.

54. Razak, I. A.; Raj, S. S. S.; Fun, H. K.; Tong, Y. X.; Lu, Z. L.; Kang, B. S. Acta Crystallogr C 2000, 56, 291.

55. Rybak-Akimova, E. V.; Nazarenko, A. Y.; Silchenko, S. S. Inorg Chem 1999, 38, 2974.

56. Cariati, F.; Erre, L.; Micera, G.; Panzanelli, A.; Ciani, G.; Sironi, A. Inorg Chim Acta 1983, 80, 57.

57. Sudbrake, C.; Muller, B.; Vahrenkamp, H. Eur J Inorg Chem 1999, 11, 2009.

58. Junk, P. C.; Smith, M. K.; Steed, J. W. Polyhedron 2001, 20, 2979.

59. Antti, B. M. Acta Chem Scand A 1976, 30, 103. 